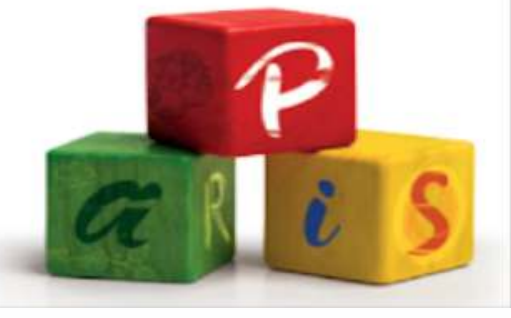

The $7^{\text {th }}$ Congress of the

EUROPEAN ACADEMY OF PAEDIATRIC SOCIETIES

October 30 - November 3, 2018, Paris, France

\title{
URINARY TRACT INFECTION: \\ A RED FLAG TO PREVENT UNINTENDED PREGNANCY IN ADOLESCENCE?
}

Denise Swei Lo¹, Eliane Roseli Barreira1 ${ }^{1}$, Itamar de Souza Santos², Alfredo Elias Gilio1

1. University Hospital of University of Sao Paulo, Department of Paediatrics, Sao Paulo, Brazil.

2. University Hospital of University of Sao Paulo, Internal Medicine Department, Sao Paulo, Brazil

\section{Background and Aims:}

Various studies have linked sexual intercourse with urinary tract infection (UTI) in women. We aim to investigate whether UTI is associated with sexual activity and increased odds of pregnancy in adolescents.

\section{Methods:}

Retrospective analysis of data from female adolescents aged 12-15 years old who visited a pediatric emergency department (ED) from 2010-2012 for suspected UTI. Patients were classified into two groups according to the results of the urine culture: UTI positive and UTI negative. We compared the occurrence of pregnancy among the two groups at the ED visit and within the following two years. Crude and age-adjusted odds ratios (aORs) with 95\% confidence interval $(\mathrm{Cl})$ were pooled when appropriate.

\section{Results:}

Of 385 female adolescents who met the study criteria, $92(23.9 \%)$ were UTI positive and $293(76.1 \%)$ were UTI negative. At the ED visit, pregnancy rates were higher among adolescents of the UTI-positive group versus those of the UTI-negative group (9.8\% vs. $2.4 \%$; aOR: $3.42 ; 95 \% \mathrm{Cl}: 1.21-10.00)$. Over the following two years, adolescents of the UTI-positive group experienced higher incidents of pregnancy compared with the UTI-negative group (14.5\% vs. $4.5 \%$; aOR: 2.89 ; $95 \%$ Cl: $1.23-6.75$ ).

Table 1. Frequencies, crude and age-adjusted odds ratios (with 95\% confidence intervals (CI)) for pregnancy according to urinary tract infection (UTI) diagnosis at baseline

\begin{tabular}{|c|c|c|c|c|}
\hline \multirow[b]{2}{*}{ Subgroup } & UTI positive & UTI negative & \multirow{2}{*}{$\begin{array}{l}\text { Crude OR } \\
(95 \% \mathrm{CI})\end{array}$} & \multirow{2}{*}{$\begin{array}{l}\text { Adjusted OR } \\
(95 \% \mathrm{CI})\end{array}$} \\
\hline & \multicolumn{2}{|c|}{ No. of events/no. at risk } & & \\
\hline Pregnancy at presentation & $9 / 92(9.8 \%)$ & $7 / 293(2.4 \%)$ & $4.43(1.60-12.74)$ & $3.42(1.21-10.00)$ \\
\hline Pregnancy during follow-up* & $12 / 83(14.5 \%)$ & $13 / 286(4.5 \%)$ & $3.55(1.53-8.16)$ & $2.89(1.23-6.75)$ \\
\hline Total pregnancies at presentation or follow-up & $21 / 92(22.8 \%)$ & $20 / 293(6.8 \%)$ & $4.04(2.07-7.90)$ & $3.27(1.65-6.53)$ \\
\hline
\end{tabular}

* (Excludes those with pregnancy at presentation.) $\mathrm{OR}=$ odds ratio.

\section{Conclusions:}

We observed increased odds of pregnancy among adolescents presenting to the ED with a confirmed UTI episode. Our findings suggest that the diagnosis of UTI in the ED represents an opportunity to detect pregnancy and to prevent further unintended pregnancy in adolescence.

\section{References:}

- Lo DS, Shieh HH, Barreira ER, Ragazzi SLB, Gilio AE. High Frequency of Staphylococcus Saprophyticus Urinary Tract Infections Among Female Adolescents. Pediatr Infect Dis J 2015;34(9):1023-5.

- Nguyen H, Weir M. Urinary tract infection as a possible marker for teenage sex. South Med J 2002;95(8):867-9.

- Finer LB, Philbin JM. Sexual initiation, contraceptive use, and pregnancy among young adolescents. Pediatrics 2013;131(5):886-91.

- Goyal M, Hersh A, Luan X, et al. Frequency of pregnancy testing among adolescent emergency department visits. Acad Emerg Med 2013;20(8):816-21.

- Vincent CR, Thomas TL, Reyes L, White CL, Canales BK, Brown MB. Symptoms and risk factors associated with first urinary tract infection in college age women: a prospective cohort study. J Urol 2013;189(3):904-10. 